\title{
Improving DC Power Supply Efficiency with Neural Network Controller
}

\author{
Weiming Li, Xiao-Hua Yu \\ Department of Electrical Engineering \\ California Polytechnic State University \\ San Luis Obispo, CA 93407, USA \\ E-mail: xhyu@ee.calpoly.edu
}

\begin{abstract}
DC-DC converters can be found in almost every power electronics device. To improve the efficiency and controller response of a DC-DC converter to dynamical system changes, neural network has been chosen as an alternative to classic methods. However, no prior work has been done in the neural network approach for control of a PSFB (Phase-Shifted Full-Bridge) converter yet. In this research, a multi-layer feedforward neural network controller is proposed. The neural network based controller has the advantage of adaptive learning ability, and can work under the situation when the input voltage and load current fluctuate. Levenberg-Marquardt backpropagation training algorithm is used in computer simulation. The neural controller is then implemented on hardware using a DSP (digital signal processor). Satisfactory experimental results are obtained.
\end{abstract}

Keywords - neural network controller, DC-DC converter, feedforward neural networks

\section{INTRODUCTION}

DC-DC converters can be found in almost every electronic device nowadays, since all semiconductor components are powered by DC sources. One of the design targets for electronic engineers is to improve the efficiency of power conversion. For PWM (pulse-width modulation) converters, switching loss is an important performance measure. Many different kinds of topologies ([1], [2], [3]) have been investigated in the past to reduce the switching loss. However, those topologies either need additional components for the power circuit, which may introduce some unstable factors to the circuit, or operate at variable frequency, which makes the filter design at output stage very difficult. Phaseshifted zero-voltage switching full-bridge converters overcome the above limitations and thus have been received more and more attention recently. It employs zero-voltageswitching (ZVS) technique and allows the voltages across the transistors to swing to zero just before the start of the next conduction cycle ([4], [5], [6]).

In general, the supply voltage and load current have a wide range of variation; so the controller has to be designed to work under such conditions. The conventional approach is to assume that the converter is operated around its equilibrium state; then a set of linear equations are derived based on this assumption [9] [10].

Artificial neural networks (ANN) have been widely used in the field of system identification, adaptive control, and statistical modelling in recent years. A neural network is composed of many non-linear adaptive processing elements and is capable of approximating any measurable function under certain conditions.

To improve the controller response of a DC-DC converter to dynamical system changes, neural network has been chosen as an alternative to classic methods ([7], [8]). However, no prior work has been done in the neural network approach for control of a PSFB (Phase-Shifted Full-Bridge) converter yet. In this research, a multi-layer feed-forward neural network controller is proposed. A Matlab Simulink model is developed first to generate training data; then the neural network is trained by Levenberg-Marquardt back-propagation algorithm. Finally, the neural controller is implemented and tested on a DSP evaluation board eZdsp® F2812 (with digital processor TMS320F2812) and a phase-shifted zero-voltageswitching circuit board UCC3895EVM. The neural network controller is fine-tuned on-line with back-propagation algorithm. Satisfactory experimental results are obtained.

\section{CIRCUIT ANALYSIS}

The circuit diagram of a PSFB DC-DC converter is shown in Fig. 1. Based on circuit analysis, the control scheme to drive the switching MOSFET (metal-oxide-semiconductor field-effect transistor, i.e., Q1, Q2, Q3 and Q4) is very complicated [6]. The circuit is operated in one of the following modes:

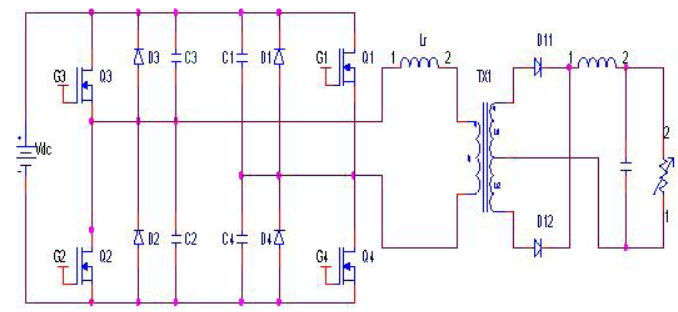

Fig. 1. PSFB DC-DC Converter

Mode 1: The diagonal MOSFET Q3 \& Q4 are conducting and power is delivered through the transformer to the load. The primary load current is flowing through the leakage inductance of the transformer. The total primary current is 
equal to the load current plus increasing magnetizing current of the transformer.

Mode 2: With Q4 on, the capacitance across Q1 is charged to $+\mathrm{V}$. When Q4 turns OFF the current through the transformer inductance starts to charge the drain source capacitance of Q4, while at the same time discharges the capacitance of Q1. This action continues until the body diode of Q1 turns ON to clamp the voltage across Q1 at approximately $0.7 \mathrm{~V}$. The current through the transformer is sustained in the upper half of the power circuit.

Mode 3: When the voltage across Q1 reaches approximately $0 \mathrm{~V}, \mathrm{Q} 1$ turns $\mathrm{ON}$. The time that is required for the capacitance of Q4 and Q1 to reach the desired voltage is determined by the characteristics of hardware. The current in this mode circulates through the conduction channels of Q3 and Q1

Mode 4: Q3 turns off. The transformer current now starts to charge and discharge the capacitance of Q3 and Q2 respectively. It again requires a finite amount of time for the drain voltage of Q2 to reach " 0 volt" at which point Q2 is allowed to be switched on. That means non-dissipate turn ON switching is accomplished.

Mode 5: With the complete discharge of its drain source capacitance Q2 is now ready to turn ON. Power is delivered to the load through the conduction path of Q1 and Q2 for an amount of time that is determined by the control circuit. Twice the product of this time, times the operating frequency of the oscillator gives the duty cycle of the converter as in a regular PWM converter. Duty Cycle D $=2 *$ Ton*Fs.

Mode 6: Following the power transfer by the above diagonal pair, Q1 turns OFF. The voltage across Q4 starts to decrease, and when this voltage reaches 0 volts the next mode starts.

Mode 7: In this mode, Q4 turns on and primary current circulates in the conduction channels of the lower pair.

Mode 8: Q2 turns OFF and current starts to charge and discharge the capacitance of Q2 and Q3 respectively. When the voltage across Q3 has reached 0 volts then Q3 turns ON non-dissipative and the complete cycle repeats itself from Mode 1

The relationship between the input/output voltage and the duty cycle can be described as:

$$
V_{0}=\left(\frac{2 n V_{i n}}{T_{s}}\right)\left(\Phi \cdot \frac{T_{s}}{2}-T_{1}-\frac{T_{2}}{2}\right)
$$

where $V_{0}$ is the output voltage, $V_{\text {in }}$ is the input voltage, both in rms value; $\mathrm{n}$ is the transformer ratio, $T_{s}$ is the switching period constant, and $\Phi$ is the duty cycle. $T_{1}$ and $T_{2}$ can be obtained from the following equations

$$
T_{1}=\frac{\left(n I+I_{c}\right) L_{r}}{V_{\text {in }}}
$$

$$
T_{2}=\frac{V_{i n}\left(C_{Q 3}+C_{Q 4}\right)}{n I}
$$

where $I$ is the load current; $C_{Q 3}$ and $C_{Q 4}$ are the capacitance of the two intrinsic capacitors of Q3 and Q4, respectively; $L_{r}$. is the resonant inductance. $I_{c}$ can be calculated as follows:

$$
I_{c}=V_{i n} \sqrt{\frac{\left(C_{Q 1}+C_{Q 2}\right)\left(J^{2}-1\right)}{L_{r}}}
$$

where

$$
J=\frac{n I}{V_{\text {in }}} \sqrt{\frac{L_{r}}{\left(C_{Q 1}+C_{Q 2}\right)}}
$$

The controller determines the duty cycle based on the input voltage and load current to achieve the desired output voltage. As shown in the above equations, the control law is highly nonlinear and as the result, it leads to a very complicated design in conventional approach.

\section{LOAD CURRENT ESTIMATION}

Based on the above the analysis, the Simulink model for load current estimation is developed and shown in Fig. 2, which illustrates the relationship between the change in load current and the change in the output voltage.

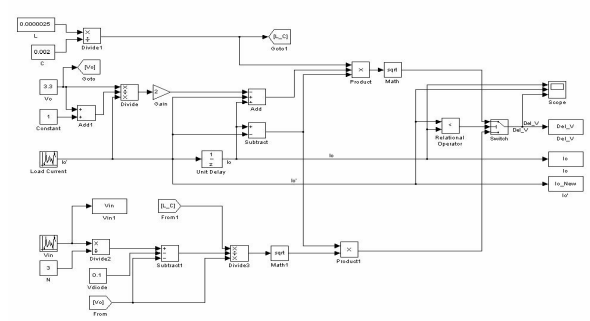

Fig. 2. Load Current Dynamic Model

It is well-known that artificial neural networks can approximate any nonlinear function to the desired accuracy, after it is fully trained. In this research, the Simulink model is used to obtain data set for the initial training of neural network. A feedforward neural network with six neurons in the hidden layer is employed. The activate function in the hidden layer is the Tangent Sigmoid function:

$$
f(x)=\frac{2}{1+e^{-2 x}}-1
$$

The weights of the neural network are initialized at random. To speed up the learning process, LevenbergMarquardt algorithm is employed:

$$
W(k+1)=W(k)+\Delta W
$$

where

$$
\Delta W=\left(J_{a}{ }^{T} J_{a}+\mu I\right)^{-1} J_{a}{ }^{T} e
$$


$J_{a}$ (also called the Jacobian matrix) is the first order derivative of the error function respect to the neural network weight, e is the output error (i.e., the difference between the neural network output and the desired output), $\mu$ is a learning parameter, and $\mathrm{k}$ is the number of iterations.

The neural network learning curve is shown in Fig. 3. From the simulation result we conclude that the neural network can successfully estimate the load current.

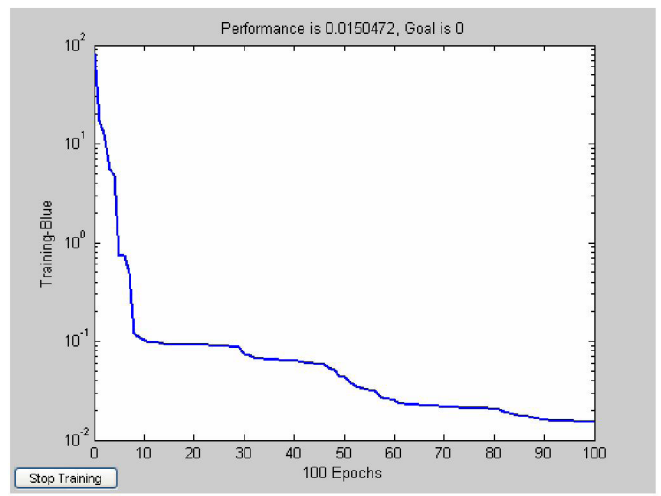

Fig. 3. Training Performance on Load Current Estimation

\section{THE NEURAL NETWORK CONTROLLER SIMULATION}

Fig. 4 describes the dynamic model for overall system, with the estimation of load current, the input voltage, and the duty cycle. A feed-forward neural network with one hidden layer is employed to calculate the desired duty cycle. The inputs to the neural network include input voltage, load current, and the change of output voltage.

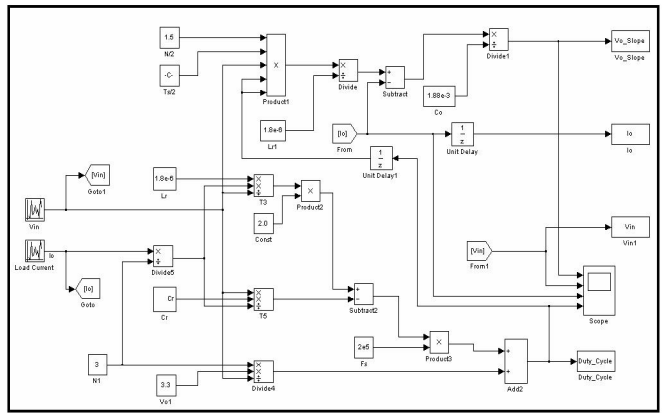

Fig. 4. The Simulink Model

There are six neurons in the hidden layer and one neuron in the output layer. The activation function for each neuron is:

$$
f(x)=\frac{1}{1+e^{-x}}
$$

The computer simulation results are shown in the following figures. Fig. 5 shows the training error is reduced to the desired accuracy after about 100 iterations. Fig. 6 demonstrates the desired value of duty cycle and the estimation of neural network controller. It is shown that the neural network can be fully trained to model the relationship between duty cycle and the change of output voltage.

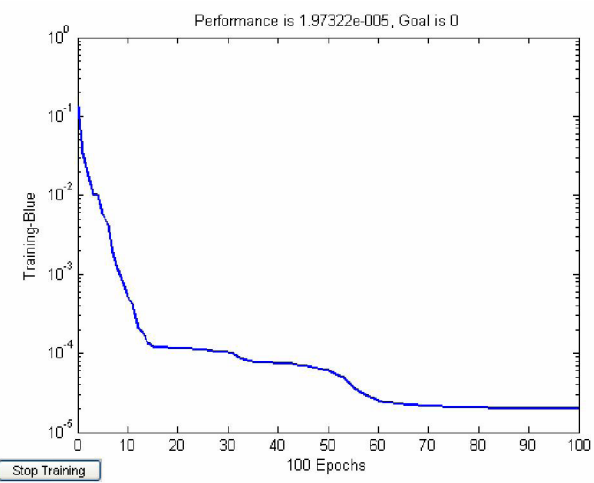

Fig. 5. Neural Network Training Error

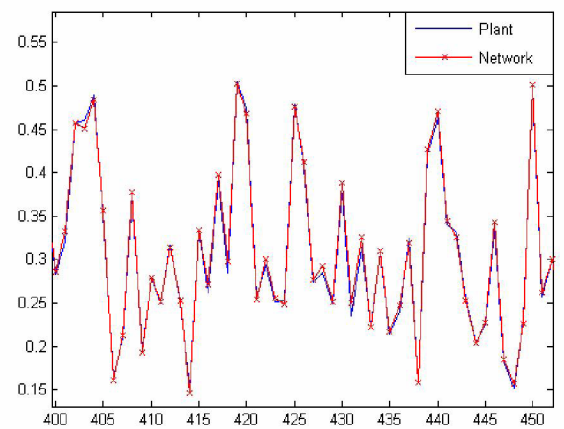

Fig. 6. Duty Cycle Estimation

\section{EXPERIMENTAL RESULTS}

In order to fully investigate the performance of the neural network controller, a DSP evaluation module eZdspß F2812 and a phase-shifted zero-voltage-switching evaluation board UCC3895EVM are used to implement the neural controller and the power circuit, respectively. The on-board digital signal processor TMS320F2812 is a 32-bit CPU with an operating speed of 150 MIPS (million instructions per second). The input voltage of the phase-shifted full-bridge converter ranges from $36 \mathrm{~V}$ to $72 \mathrm{~V}$, with $48 \mathrm{~V}$ as its nominal input. The nominal value of the output of the converter is $3.3 \mathrm{~V}$, with nominal load current. The neural network controller is developed in $\mathrm{C}$ language. The overall system schematic is shown in Fig. 8 (next page).

First, the analog signals from the power circuit are digitized and processed before being fed into the neural 
network's inputs. Since the power circuit is driven by 400 $\mathrm{kHz}$ high frequency PWM signals, the raw output voltage signal is quite noisy during the switch turn-on and turn-off time. To solve this problem, multiple samples are taken to obtain the average value over a certain period of time. Next, the processed data is fed into the neural network to estimate the desired duty cycle. The new estimated duty cycle is then applied to control the power circuit, and the output voltage is monitored.

At nominal input voltage $48 \mathrm{~V}$, the $10 \%$ to $90 \%$ load step up and $90 \%$ to $10 \%$ load step down transient response of the overall system is captured on the oscilloscope as shown in Fig. 7. The difference between the voltage peak and the nominal output is about 0.25 to $0.3 \mathrm{~V}$ for both load step up and step down transient response, which is well within the normal range of switching regulator's transient performance. However, it is observed that the converter's response time to load transient is longer than the case of analog controller. This is due to the on-line training and long processing time for floating-point format running on a fixed-point microprocessor.

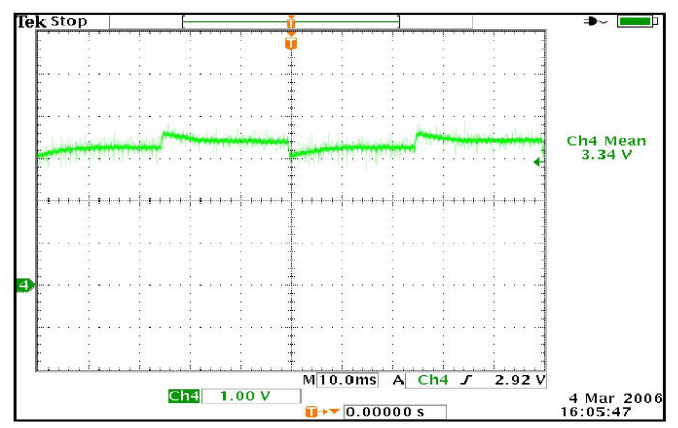

Fig. 7. $10 \% \leftarrow \rightarrow 90 \%$ Load Transient Response at $48 \mathrm{~V}$ with Neural Network Controller

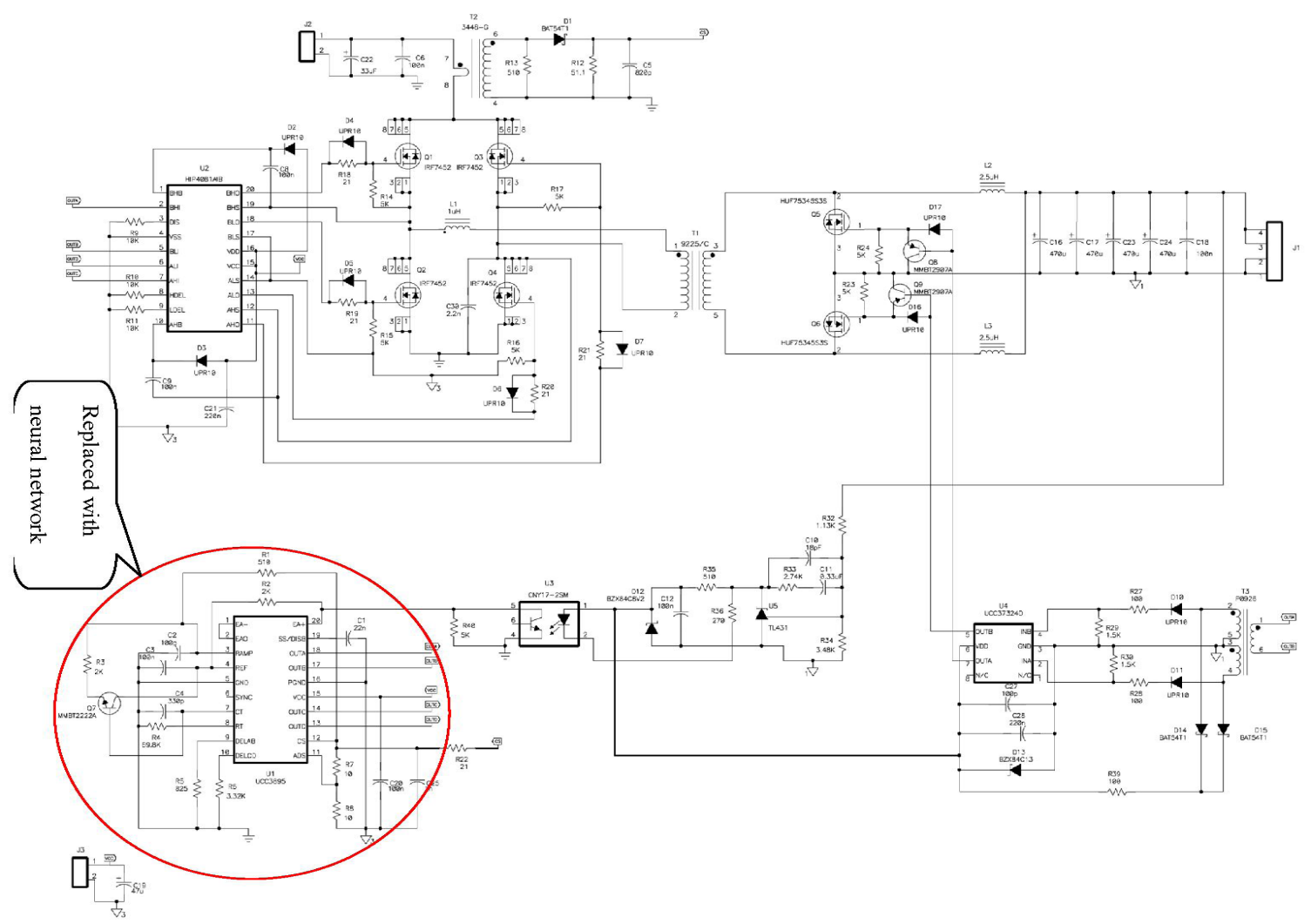

Fig. 8. Power Circuit and Controller Schematic 
Another test is performed when the input voltage is $60 \mathrm{~V}$. The $10 \%$ to $90 \%$ load step up and $90 \%$ to $10 \%$ load step down transient response of the overall system is shown in Fig. 9. The difference between the voltage peak and the nominal output is about 0.4 to $0.5 \mathrm{~V}$ for transient response. In both cases ( $48 \mathrm{~V}$ and $60 \mathrm{~V}$ input voltage), when the load changes, the neural network controller successfully generates the control (i. e., duty cycle) to keep the output voltage stable, with the desired level.

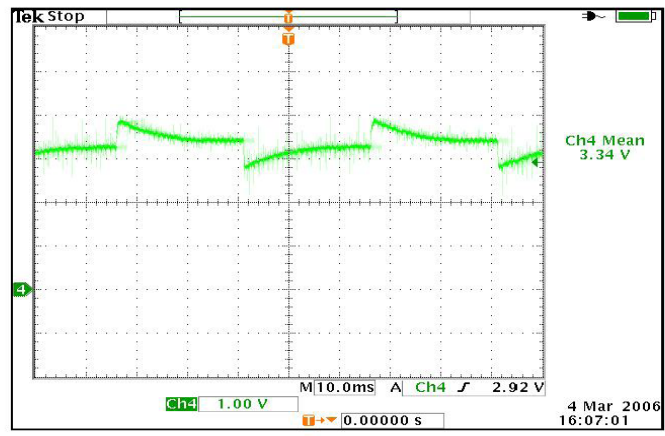

Fig. 9. $10 \% \leftrightarrow 90 \%$ Load Transient Response at $60 \mathrm{~V}$ with neural network controller

It is known that phase-shifted zero-voltage switching full-bridge converters employ zero-voltage-switching (ZVS) technique which allows the voltage across each transistor to swing to zero just before the start of the next conduction cycle ([4], [5], [6]) to reduce power consumption and thus improve efficiency. This approach can also minimize the switching stress on the transistor so that the longevity of the device is extended. Fig. 10 shows the oscilloscope capture of switching waveform of MOSFET Q2 in the power circuit, where $\mathrm{V}_{\mathrm{g}}$ is the driving signal at the gate terminal and $V_{\mathrm{ds}}$ is the voltage across drain and source, respectively. The waveforms clearly show that the voltage across the MOSFET swings to zero before the driving signal is asserted. Therefore, the transistor is turned on with zero cross voltage and the switching loss is greatly reduced. Similar waveforms can be captured for other MOSFETs in the converter.

Finally, the performance of on-line training algorithm is investigated. The output voltages at varying load conditions (with nominal input voltage) are measured and compared, as shown in Fig. 11. The off-line training is based on an ideal Simulink model (without considering the voltage drop and power consumption of each circuit element); therefore the resulting output voltage is significantly lower than the desired value. With the correction of on-line training, the performance of neural network controller is significantly improved and performs as expected.

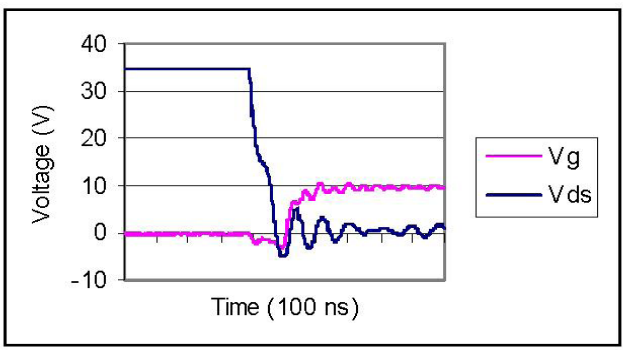

Fig. 10. Zero-Voltage Switching

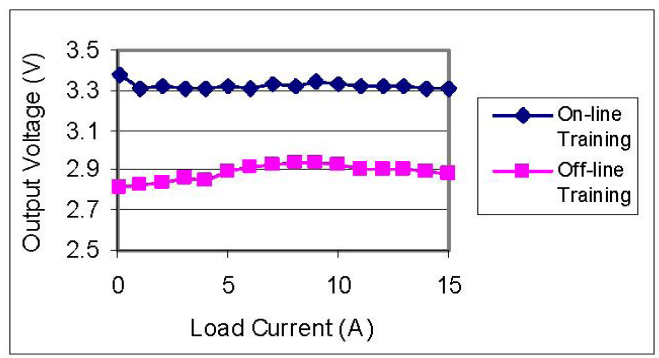

Fig. 11. On-line vs. Off-line Training

\section{CONCLUSION}

A neural controller as an alternative to classic controller for PSFB DC-DC converter is proposed in this paper. Both simulation and experimental results show that the neural network controller is able to estimate the desired duty cycle under several dynamic conditions. More research works will be done to optimize the software to speed up the neural controller for real-time applications.

\section{ACKNOWLEDGEMENTS}

This work was partially sponsored by the Department of the Navy, Office of Naval Research, under Award \# N00014-06-1-1111. Also, the authors would like to thank Dr. Taufik for providing the hardware.

\section{REFERENCES}

[1] P. R. Chetty, "Resonant power supplies: Their history and status," IEEE Aerosp. Electron. Syst. Mag, vol. 7, no. 4, pp. 23-29, Apr. 1992

[2] M. G. Kim and M. J. Youn, "An energy feedback control of series resonant converters," IEEE Trans. Power Electron, vol. 6, no. 4, pp. 338-345, Jul. 1991

[3] J M Carrasco, E Galván, G E Valderrama R Ortega, and A Stankovic, "Analysis and experimentation of nonlinear adaptive controllers for the series resonant converter," IEEE Trans. Power Electron., vol. 15 , no. 3 , pp. 536-544, May 2000

[4] H.-S. Choi and B. H. Cho, "Novel zero-current-switching (ZCS) PWM switch cell minimizing additional conduction loss," IEEE Trans. On Industrial Electronics, Vol. 49, No. 1, P. 165-172, February 2002. 
[5] H.-S. Choi, J.-W. Kim, and B. H. Cho, "Novel zero-voltage and zerocurrent-switching (ZVZCS) full-bridge PWM converter using coupled output inductor," IEEE Trans. On Power Electronics, Vol. 17, No. 5, P. 641-648, September 2002.

[6] X. Ruan and Y. Yan, "A novel zero-voltage and zero-current-switching PWM full-bridge converter using two diodes in series with the lagging leg," IEEE Trans. On Industrial Electronics, Vol. 48, No. 4, P. 777-785, Augu st 2001.

[7] J. M. Quero, J. M. Carrasco, and L. G. Franquelo, "Implementation of a neural controller for the series resonant converter," IEEE Trans. On Industrial Electronics, Vol. 49, No. 3, P. 628-639, June 2002.

[8] F. Kamran, R. G. Harley, B. Burton, T. G. Habetler, and M. A. Brooke, "A fast on-line neural-network training algorithm for a rectifier regulator," IEEE Trans. On Power Electronics, Vol. 13, No. 2, March 1998.

[9] B. Choi, J. Kim, B. H. Cho, S. Choi, and C. M. Wildrick, "Designing control loop for DC-to-DC converters loaded with unknown AC dynamics," IEEE Trans. On Industrial Electronics, Vol. 49, No. 4, P. 925-932, August 2002

[10] Texas Instrument Inc, "BiCMOS advanced phase shift PWM controller," Data sheet of UCC3895, January 2001. 